\title{
Avifauna de la Reserva de la Biosfera Barranca de Metztitlán, Hidalgo, México
}

\author{
Birds of the Biosphere Reserve Barranca de Metztitlán, Hidalgo, Mexico
}

\author{
Raúl Ortiz-Pulido ${ }^{*}$, Jessica Bravo-Cadena ${ }^{1}$, Vanessa Martínez-García ${ }^{1}$, Doroteo Reyes ${ }^{1}$, Ma. Eugenia \\ Mendiola-González², Guillermo Sánchez ${ }^{3}$ y Maximino Sánchez ${ }^{2,3}$
}

${ }^{1}$ Laboratorio de Ecología de Poblaciones, Centro de Investigaciones Biológicas, Universidad Autónoma del Estado de Hidalgo. Apartado postal 69, Pachuca 42001, Hidalgo, México.

Dirección actual: Laboratorio de Ecología del Comportamiento, Centro Tlaxcala de Biología de la Conducta UAT-UNAM. Carretera Tlaxcala-

Puebla Km. 1.5, Colonia Xicotencatl 90070, Tlaxcala, Tlaxcala, México.

${ }^{2}$ Dirección de la Reserva de la Biosfera Barranca de Metztitlán. Domicilio conocido, Metztitlán, Hidalgo, México.

${ }^{3}$ Domicilio conocido. Venados, Barranca de Metztitlán, Hidalgo, México.

*Correspondencia: raulortizpulido@yahoo.com

\begin{abstract}
Resumen. El manejo y conservación de un área natural protegida depende en gran parte del conocimiento biológico que se tenga sobre ella. En este estudio se presenta el listado de las aves de la Reserva de la Biosfera Barranca de Metztitlán, Hidalgo, México realizado durante 7 años de trabajo de campo y que incluye 271 especies. Las familias más ricas fueron Tyrannidae y Parulidae (24 especies cada una), Emberizidae (19), Icteridae (13) y Trochilidae (12). Se registran 117 especies como residentes, 88 migratorias, 34 ocasionales, 6 con poblaciones residentes-migratorias y 26 sin estacionalidad clara. Se observaron 16 especies abundantes, 67 comunes, 153 raras y 35 sin abundancia relativa clara. Los tipos de vegetación más utilizados por las aves son: matorral submontano (113 especies), bosque tropical caducifolio (97), bosque de tascate (96) y matorral crasicaule con dominancia de $S$. dumortieri (91). Los gremios alimenticios mejor representados fueron: insectívoro (235 especies), frugívoro (88) y granívoro (85). Con base en la normatividad mexicana, se registraron 17 especies bajo alguna categoría de riesgo y 32 con algún grado de endemismo. En la zona habitan cerca del $60 \%$ de las aves de Hidalgo y $27 \%$ de las de México, razón por la cual se sugiere que esta zona sea declarada Área Importante para la Conservación de las Aves (AICA) en México.
\end{abstract}

Palabras clave: listado de aves, inventario avifaunístico, Barranca de Metztitlán, Hidalgo, Reserva de la Biosfera.

\begin{abstract}
Management and conservation of natural protected areas depends critically on their biological knowledge. Herein we report a check-list of the Barranca de Metztitlán Biosphere Reserve, Hidalgo, Mexico. We registered 271 species. The families that include more species were Tyrannidae and Parulidae (24 species each one), Emberizidae (19), Icteridae (13) and Trochilidae (12). We recorded 117 resident species, 88 migratory, 34 transient, 6 with residentmigratory populations and 26 with status not clear. We registered 16 abundant species, 67 common, 153 rare, and 35 with undetermined abundance. The richest vegetation types were: submontane shrubland with 113 species, tropical deciduous forest with 97, Juniperus forest with 96, and crasicaule shrubland with Stenocereus dumortieri dominance with 91. The best represented gremials were: insectivorous (235 species), frugivorous (88), and granivorous (85). We found 17 species under some risk category according with the Mexican law, and 32 species possesses some endemic degree. In the reserve inhabit near of the $60 \%$ of the birds of the Hidalgo state and $27 \%$ of the Mexican birds. This region should be considered as an Important Bird Area in Mexico.
\end{abstract}

Key words: bird check-list, bird inventories, Barranca de Metztitlán, Hidalgo, Biosphere Reserve.

\section{Introducción}

El funcionamiento exitoso de un área natural protegida depende en gran medida de los conocimientos que se tengan sobre las especies de animales presentes en ella. Sin esta información se pueden cometer errores al

Recibido: 04 mayo 2009; aceptado: 13 octubre 2009 determinar las áreas biológicamente más diversas o con mayores endemismos. Por tanto, es necesario conocer la ubicación espacial de los hábitats o tipos de vegetación que usa cada especie animal en estas áreas, así como la presencia temporal de cada especie en ellos, ya que con esta información se puede planear la conservación biológica en diversos niveles espaciales (local, municipal, regional, estatal, nacional) y temporales (años, décadas). 
Los datos sobre la localización de las especies animales constituyen también la base sobre la cual se pueden establecer estrategias que permiten detectar las zonas más adecuadas para realizar actividades de interés humano, como son las de conservación, investigación, extracción y/o producción de alimentos.

Para Hidalgo, México, recientemente se ha publicado el primer listado estatal global de las especies de aves presentes (Martínez-Morales et al., 2007). Sin embargo, los estudios han sido limitados; por ejemplo, hasta finales del siglo XX sólo se habían registrado 13 estudios relacionados con este grupo en la zona (Mancilla, 1988), a la fecha se cuenta con varias listas (e.g., Villada, 1865; Martín del Campo, 1936, 1937; Friedman et al., 1950; Cantú, 1953; Arellano y Rojas, 1956; Miller et al., 1957; Bjelland y Ray, 1977; Sánchez, 1978; Dirección General de Fauna Silvestre, 1980; Pichardo, 1987; Mancilla, 1988; Rojas-Soto et al., 2002; Hernández-Avilés et al. 2003; Arregui, 2004; Martínez-Morales, 2004; Mauricio-López, 2005; Martínez-García, 2006; Díaz-Valenzuela, 2008; Ortiz-Pulido et al., 2006, 2008) y recopilaciones parciales (Villavicencio et al., 1993).

Para la Reserva de la Biosfera Barranca de Metztitlán (RBBM), Hidalgo, esta situación no es diferente, sólo existen recopilaciones parciales o extrapolaciones bibliográficas de las especies de aves presentes, y no se conoce su estacionalidad ni los tipos de vegetación que usa cada una. La RBBM está localizada en la depresión profunda de una llanura que antiguamente se llamó Barranca Grande (Villada, 1865). Esta reserva presenta al menos 6 tipos de vegetación, principalmente xerófitos (CONANP, 2003). Se sabe que los pobladores prehispánicos hacían uso intensivo de algunas especies de aves de la zona (Villada, 1865; Cuevas, 2002) y que existió un conocimiento extenso sobre ellas. No obstante, la información sistemática más antigua para aves con la que se cuenta fue elaborada a mediados del siglo XIX e indica la presencia de 31 especies (Villada, 1865). A finales del siglo XX se publicaron 2 listados nuevos de aves para esta zona; el primero, curiosamente contenido en un artículo de botánica, registra 93 especies, enfocándose en las aves que habitan La Vega, una zona afectada por la actividad humana (Sánchez, 1978); el segundo se enfoca en ambientes más prístinos; registra 163 especies de aves presentes en 3 áreas, de las cuales 2 se encuentran en los alrededores de la zona actualmente ocupada por la RBBM (Mancilla, 1988). A fines del siglo pasado y principios del XXI han aumentado los trabajos en la zona; por ejemplo, Ramírez et al. (1994) publicaron el primer registro de Gavia immer para la laguna de Metztitlán. La Dirección de la RBBM (CONANP, 2003) publicó un reporte técnico que no fue verificado en campo y con algunos registros erróneos, donde se indica la presencia de 215 especies. Ortiz-Pulido et al. (2006) publicaron las fichas técnicas de las 11 especies de colibríes presentes, mientras que Martínez-García (2006) registró los colibríes presentes en 4 tipos de vegetación. Finalmente, Ortiz-Pulido y Martínez-García (2006) describieron una hembra anidante de Calothorax lucifer con plumaje de macho hallada en el área, especie previamente reportada poéticamente en la zona (Hernández-Avilés et al., 2003). A pesar del incremento reciente en las publicaciones dedicadas a la avifauna de la zona, no hay un listado de aves actualizado para la reserva.

El objetivo de este trabajo es describir las especies de aves presentes en la RBBM, indicando para cada una su estacionalidad, abundancia, los hábitats o tipos de vegetación que usan, su alimentación, y el grado de conservación y endemismo. Con ello se pretende contribuir al conocimiento ornitológico de esta región poco estudiada, facilitar y estimular la realización de otros estudios de aves en la región, y dar información que pueda ayudar a planear la conservación de este tipo de fauna en la RBBM.

\section{Materiales y métodos}

La RBBM tiene una extensión de $960.42 \mathrm{~km}^{2}$ y está localizada en el centro este del Estado de Hidalgo, México

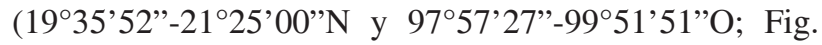
1). Presenta una topografía accidentada y un gradiente altitudinal que va desde los $1100 \mathrm{~m}$ snm hasta los 2600 $\mathrm{m}$ snm, lo que determina la presencia de diversos climas y tipos de vegetación (CONANP, 2003). El clima varía de seco semicálido a seco templado, con temperatura promedio anual de $18-22^{\circ} \mathrm{C}$ y una precipitación media anual de 400-700 mm (CONANP, 2003). Esta área constituye un corredor biológico entre la vegetación neártica de la zona norte de México y la vegetación tropical del altiplano central de México (CONANP, 2003). En la zona están presentes corrientes superficiales de agua, estacionales y continuas (e.g., río Venados), así como una laguna (CONANP, 2003).

La flora vascular registrada de la RBBM está constituida por 465 especies pertenecientes a 270 géneros y 83 familias que se localizan en 6 tipos principales de vegetación: matorral xerófilo (que se divide en 4 subtipos, véase abajo), matorral submontano, bosque tropical caducifolio, bosque de coníferas (también dividido en 4 subtipos, véase abajo), pastizal y vegetación ribereña (dividida en 2 subtipos, véase abajo) (CONANP, 2003). El trabajo de campo fue realizado especialmente en los 4 primeros tipos de vegetación (en los otros 2 se realizaron observaciones casuales), por ser éstos de origen natural, 


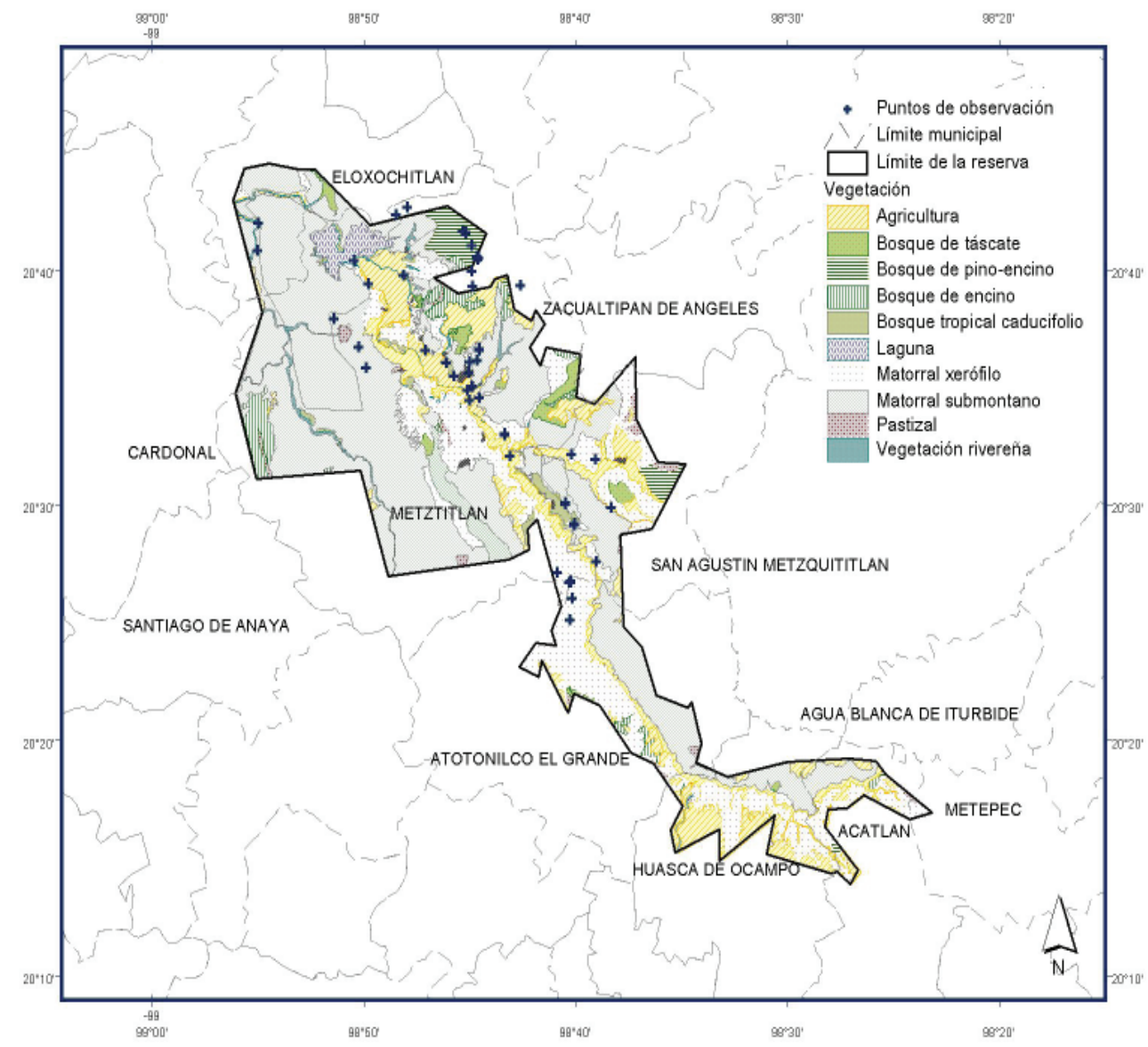

Figura 1. Localización de la Reserva de la Biosfera Barranca de Metztitlán, en Hidalgo, México. Se presentan con diferente relleno los principales tipos de vegetación localizados en la zona. Se indican los sitios que fueron más visitados durante este estudio, que son representativos de los diferentes tipos de vegetación presentes en la reserva.

porque cuentan con la mayor área en la RBBM y son los que menos influencia humana tienen (CONANP, 2003). Los 2 primeros tipos de vegetación cubren el $68.72 \%$ de la superficie de la RBBM, mientras que el bosque tropical caducifolio cubre el $1.27 \%$. Las áreas cubiertas por estos 3 tipos de vegetación están consideradas como las zonas núcleo de la reserva (CONANP, 2003). Los bosques de coníferas se localizan al noreste de la RBBM, en las partes altas de las montañas, y constituyen el $7.44 \%$ del área de la reserva (CONANP, 2003).

El matorral xerófilo presente en la RBBM, localizado desde los 1100 hasta los $1750 \mathrm{~m}$ snm, puede dividirse en 4 subtipos estructuralmente diferenciados (usando parcialmente la clasificación de Rzedowski, 2005); 1) con dominancia de Cephalocereus senilis (Cactaceae); 2) con dominancia de Stenocereus dumortieri (Cactaceae); 3) con dominancia de Myrtillocactus geometrizans (Cactaceae), y 4) con dominancia de Opuntia sp. (Cactaceae). En este trabajo se emplea esta subdivisión, ya que la estructura de la vegetación de cada uno está determinada por la dominancia de una u otra especie de planta, y se sabe que la estructura de la vegetación está relacionada con la riqueza de aves (Cueto y Lopéz, 1999), tal vez porque un sitio con mayor estructura ofrece más condiciones y recursos (como sitios de percha, frutos o insectos). Esta subdivisión se consideró porque en zonas desérticas, como la de esta zona, se ha encontrado una relación entre la estructura de la vegetación y la riqueza de aves (Whitmore, 1975; Beedy, 1981; Anderson et al., 1983) y no considerar este hecho enmascararía patrones en la riqueza que se registra para cada tipo de vegetación.

Los subtipos primero y cuarto de matorral xerófito presentan 2 estratos: arbóreo de 5 a $10 \mathrm{~m}$ y herbáceo, comúnmente menor a $1 \mathrm{~m}$ de altura. El segundo subtipo de vegetación presenta 3 estratos: arbóreo de 5 a $6 \mathrm{~m}$, arbustivo de 1 a $3 \mathrm{~m}$ y herbáceo, comúnmente menor a un $1 \mathrm{~m}$ de 
altura (CONANP, 2003). El tercero, matorral crasicaule, es un subtipo de vegetación abierta, caracterizado por la dominancia de especies crasicaules, como M. geometrizans. Esta especie merece especial atención pues tiene muchas ramificaciones en su tallo y una gran producción de frutos, lo que facilita su uso por parte de las aves. Sus frutos son pequeños, de color purpúra; florece y fructifica entre abril y julio, mezclándose con especies arbustivas del género Opuntia y herbáceas anuales y perennes (CONANP, 2003).

El matorral submontano (que corresponde al matorral crasicaule de Rzedowski, 2005), se encuentra entre los 1 600-1 $800 \mathrm{~m}$ snm. La estructura de este tipo de vegetación se caracteriza por la dominancia del estrato arbustivo que cubre $80-90 \%$ del área y que alcanza una altura máxima de $2 \mathrm{~m}$, aunque también se localizan individuos de Yuca sp. y de M. geometrizans de cerca de 6 $\mathrm{m}$ de altura dispersos en el paisaje (CONANP, 2003).

El bosque tropical caducifolio se presenta entre los 1 100-1 $500 \mathrm{~m}$ snm. Presenta 3 estratos: arbóreo de 8 a $12 \mathrm{~m}$, arbustivo de 0.9 a 2.2 m y herbáceo. El primero está dominado por la especie Bursera morelensis (Burseraceae) que da una fisonomía particular a este tipo de vegetación. Esta especie se caracteriza por ramificarse a baja altura, presentando copas comúnmente más anchas que altas, y por tener corteza rojiza y no presentar hojas durante 6 o 7 meses, razón por la cual el bosque tropical caducifolio muestra un aspecto seco y rojizo durante gran parte del año. Prosopis laevigata (Fabaceae) se encuentra en la parte baja de los cerros dominados por este tipo de vegetación (CONANP, 2003).

Los bosques templados se localizan entre los 1 650-2 $600 \mathrm{~m}$ snm. En la RBBM se pueden dividir en 4 subtipos diferentes: bosque de tascate (con dominancia de Juniperus sp., Cupressaceae), bosque de encino (Quercus sp., Fagaceae), bosque de pino-encino (Quercus sp. y Pinus sp., Pinaceae) y bosque de pino (Pinus sp., Pinaceae). El bosque de tascate se localiza por debajo de los 1600 $\mathrm{m}$, en una franja que llega hasta los límites de matorral submontano o del matorral xerófilo. El bosque de encino se localiza entre los 1 600-1 800 m snm y está dominado por especies del género Quercus que presentan alturas de 6-14 m, con un estrato arbustivo escaso y poco estrato herbáceo. Los bosques de encino, pino y pino-encino, con dominancia de los géneros Quercus y Pinus, con alturas entre 12 y $18 \mathrm{~m}$ para encino y más de $20 \mathrm{~m}$ para pino, se localizan por arriba de los $1800 \mathrm{~m}$ snm (CONANP, 2003).

La vegetación ribereña puede dividirse en 2 , la localizada en La Vega (cerca de los 1100 m snm), que presenta una perturbación humana intensa, y aquella localizada a lo largo de los causes de ríos temporales (comúnmente $>1150 \mathrm{~m} \mathrm{snm}$ ), sobre las laderas de la barranca, con poca perturbación humana.

Para la determinación de la avifauna presente en cada uno de los tipos de vegetación indicados arriba, se reunió la información correspondiente a registros de aves realizados por los autores y colaboradores desde el 2002 al 2008, así como los registros bibliográficos (véase Introducción). Los registros realizados en este estudio se basaron en transectos (terrestres y acuáticos, con más de 500 horas de observación), captura con redes (cerca de 1100 horas red), llamados con grabadora (en el caso de búhos, cerca de 30 noches) y observaciones casuales (esfuerzo no medido, pero a lo largo de 4 años por 3 observadores). En general, el esfuerzo de muestreo por tipo de vegetación fue ad libitum, pero en algunos de ellos se hicieron muestreos sistemáticos. Para matorral submontano, bosque de encino, bosque de pino-encino, matorral crasicaule con dominancia de $S$. dumortieri, matorral crasicaule con dominancia en M. geometrizans y bosque tropical caducifolio se recorrió un transecto de $1 \mathrm{~km}$ de largo por 40 de ancho, por mes, durante un año (entre 2002 y 2005) en cada tipo de vegetación, siguiendo la técnica sugerida por Emlen 1971. Para bosque tropical caducifolio, matorral submontano, matorral crasicaule con dominancia de C. senilis y matorral crasicaule con dominancia de $S$. dumortieri se muestrearon 70 puntos de conteo (de septiembre a diciembre 2007); siguiendo la técnica indicada por Bibby et al., 2000) y se colocaron 6-7 redes ornitológicas (de $12 \times 3 \mathrm{~m}$ de $35 \mathrm{~mm}$ de apertura), 2 días de cada mes, de enero a marzo de 2008, por tipo de vegetación. Los sitios más visitados en la RBBM durante el muestreo se presentan en la figura1. Las observaciones se realizaron comúnmente media hora antes de la salida del sol hasta media hora después de la puesta del sol (además, se realizaron observaciones en la noche para el registro de especies nocturnas). Todas las aves vistas durante las observaciones fueron registradas. Para determinar las especies en campo se usaron binoculares $(8 \times 40$ y $10 \times 50 \mathrm{~mm})$, guías de identificación de aves y textos especializados (Peterson y Chalif, 1989; Howell y Webb, 1995; Johnsgard, 1997; Del Hoyo et al., 1999; Williamson, 2001; Howell, 2002; National Geographic Society, 2002). De manera no sistemática se realizaron observaciones en las orillas del río Venados y la Vega de Metztitlán, registrando las especies observadas. Los nombres científicos siguen la taxonomía propuesta por AOU $(1998,2000)$ y suplementos (Banks et al., 2002, 2003, 2004, 2005, 2006, 2007, 2008).

Para hacer comparables los valores de abundancia con los de trabajos futuros similares (en donde se defina claramente el esfuerzo realizado), se usó un método, modificación del planteado por Emlen (1971), que se denominó esfuerzo de muestreo estandarizado (EME). 
Este nuevo método consiste en dar una categoría de abundancia a las especies dependiendo del número de registros por especie en 100 horas de observación o 400 ha muestreadas (en una hora de observación se muestrean aproximadamente 4 ha, si se recorre $1 \mathrm{~km}$ y se observan todas las aves presentes en $20 \mathrm{~m}$, a cada lado, durante el recorrido; véase Emlen, 1971). A las especies registradas se les asignó 1 de 4 categorías de abundancia relativa. Una especie fue considerada rara, si se registró $<3.5$ veces en un EME; común, si tuvo entre 3.5 y 21 registros en un EME; abundante, si tuvo > 22 registros en un EME, y; de abundancia indeterminada cuando se careció de información directa de campo para catalogarla. Estas tasas se obtienen de dividir el número de registros de una especie entre las horas muestreadas y multiplicando el resultado por 100 horas. Si se usan hectáreas, se divide el número de registros entre las hectáreas muestreadas y se multiplica por 400 hectáreas. Los valores límite que aquí se proponen para diferenciar entre categorías de abundancia (i.e., 3.5 y 21 registros) dan como resultado que en una comunidad de aves del 5 al $10 \%$ de las especies sean abundantes, del 15 al $30 \%$ comunes y del 60 al $75 \%$ raras, una distribución de abundancia de especies descrita generalmente en estudios de comunidades (Tokeshi, 1993). Considerando lo anterior, los resultados del método EME reflejan la distribución de abundancias de las especies encontrada en comunidades naturales (Begon et al. 2006) y son representativos de las abundancias relativas reales.

Para determinar la estacionalidad, se consideraron los datos obtenidos durante este estudio o en su defecto lo registrado bibliográficamente (sensu Howell y Webb, 1995). Para ello, cada especie se asignó en 1 de 4 categorías: 1) migratoria, la que no se reproduce en la zona pero puede ser observada durante varios meses, especialmente durante el invierno; 2) residente, la que se reproduce en el área y puede ser observada durante algunos meses o todo el año; 3) ocasional, la que no se reproduce en el área, pero puede ser observada ocasionalmente en la zona, en meses no siempre consecutivos, y 4) indeterminada, especie para la que no existen datos suficientes para determinar su estatus en la zona (Howell y Webb, 1995; Johnsgard, 1997).

Para determinar el tipo de vegetación que cada especie usa, se consideró el sitio en que se registró a cada una durante este estudio, o lo registrado bibliográficamente para la RBBM (Villada, 1865; Sánchez, 1978; Mancilla, 1988). En este caso se determinaron las siguientes categorías: bosque tropical caducifolio, matorral xerófito con dominancia de $M$. geometrizans, matorral xerófito con dominancia de $S$. dumortieri, matorral xerófito con dominancia de Cephalocereus senilis, matorral xerófito con dominancia de Opuntia sp., matorral submontano, bosque de tascate, bosque de pino-encino, bosque de pino, bosque de encino, vegetación ribereña, zona de transición entre bosque de táscate-matorral xerófito-matorral submontano, pastizal, zona perturbada de La Vega y hábitat no determinado. La última categoría se aplicó cuando los autores que registran la especie no indicaron el hábitat. Para las especies que no usan estos tipos de vegetación se consideraron sus hábitos acuáticos o aéreos.

La alimentación de cada especie de ave fue determinada tomando en cuenta información bibliográfica (Del Hoyo et al., 1992, 1996, 1999, 2005; Ortiz-Pulido et al., 1995, 2001; Arizmendi y Espinosa de los Monteros, 1996; Garza et al., 2004) o, cuando fue posible, con observaciones directas en campo. A cada especie se asignó al menos una de las siguientes 8 categorías de alimentación: carroñera, carnívora, granívora, frugívora, nectarívora, omnívora (que consume diferentes recursos, como semillas, insectos, néctar, vertebrados, frutos, pétalos y hojas), consumidora de vegetación acuática y consumidora de invertebrados.

A las especies de distribución restringida se les asignó un grado de endemismo (sensu González-García y Gómez de Silva, 2003). Se consideró endémica de México, la especie que está circunscrita a los límites políticos del país; cuasiendémica, aquella cuya distribución se extiende fuera de México en un área no mayor de $35000 \mathrm{~km}^{2}$, y semiendémica, la que es endémica de México o de una región del país durante una época del año. Las categorías de riesgo, amenazada y bajo protección especial, se definieron con base en SEMARNAT (2002).

Para completar la lista de especies de aves presentes en la RBBM se examinaron los principales estudios publicados (Villada, 1865; Sánchez, 1978; Mancilla 1988), haciendo hincapié en que la identidad de las especies mencionadas por Villada (1865) fue actualizada a la taxonomía contemporánea por Adolfo NavarroSigüenza (Cuevas, 2002). La presencia en la RBBM de especies dudosas registradas en esos estudios se evaluó de acuerdo con la distribución actual conocida (sensu Howell y Webb, 1995). Las especies registradas previamente, cuya distribución hipotética cae en la RBBM (sensu Howell y Webb, 1995), y que no fueron registradas en el trabajo de campo se integraron al listado que aquí se presenta.

\section{Resultados}

En la RBBM se han registrado 271 especies de aves, pertenecientes a 54 familias y 17 órdenes (Apéndice 1). Las familias mejor representadas fueron: Tyrannidae y Parulidae (24 especies cada una), Emberizidae (19), Icteridae (13) y Trochilidae (12). Del total de especies 117 son residentes, 88 migratorias, 34 ocasionales, 6 tienen poblaciones residentes y migratorias; para 23 no fue 
posible determinar su estacionalidad y de 3 no está claro si presentan poblaciones residentes o migratorias o ambas (una de ellas fue registrada por otros autores y no hay más datos; véase Apéndice 1).

En cuanto a la abundancia relativa, 16 son abundantes, 67 comunes, 153 raras. Para 35 especies no se pudo determinar su abundancia relativa por haberse registrado en otros trabajos o no tener suficientes registros (el caso de las aves de hábitos acuáticos o aéreos).

La zona perturbada de La Vega es el tipo de vegetación más usado por las especies (119), seguida por el matorral submontano (113; Fig. 2). La mayoría usa 1 o 2 tipos de vegetación (84 y 55 especies, respectivamente) y pocas más de 3 (Fig. 3). Para 2 taxa (Glaucidium gnoma y Sialia sp.) no fue posible determinar el tipo de vegetación usado; ambas fueron registradas previamente por otros autores en la zona y no fue posible definir la zona en que las registraron.

Los gremios de alimentación que agruparon mayor número de especies fueron el insectívoro (235), el frugívoro (88) y el granívoro (85) (Fig. 4). La mayoría de las especies consume menos de 2 categorías de alimento (Fig. 5).

Por grado de conservación, 17 especies quedaron anotadas en alguna categoría de riesgo; 5 amenazadas y 12 bajo protección especial (Apéndice 1). Por grado de endemismo se anotaron 8 especies endémicas, 5 cuasiendémicas y 19 semiendémicas (Apéndice 1).

\section{Discusión}

El análisis de los resultados permite sugerir que la RBBM es un sitio importante de conservar en el nivel regional, estatal y nacional, desde el punto de vista avifaunístico; este sitio representa un lugar donde se podría proteger gran parte de la avifauna estatal de Hidalgo. Considerando que en este estado se ha corroborado la presencia de 468 especies de aves (Martínez-Morales et al., 2007; Valencia-Hervert et al., 2008, 2009), el número de las presentes en la RBBM constituye el $59.9 \%$ de la avifauna del estado. Dada su riqueza, la RBBM debería considerarse Área Importante para la Conservación de las Aves (Arizmendi y Valdelamar, 1999), ya que en México se han registrado 1018 especies (Peterson y Chalif, 1989; pero véase también Navarro-Sigüenza y Peterson, 2004, quienes sugieren más de 1200) y más del $26.7 \%$ de ellas se localizan en la RBBM.

Independientemente de su importancia regional, estatal y nacional, la RBBM se constituye también como un sitio biológicamente diverso en comparación con otras zonas similares, algunas de ellas de mayor área. Tomando en cuenta sólo ambientes desérticos de México y de los Estados Unidos de América, la RBBM tiene una riqueza ornitológica alta. Por ejemplo, en las Reservas de la Biosfera Tehuacán-Cuicatlán (que ocupa 10000 km²) y La Michilia (700 km², en el desierto chihuahuense), y en los desierto del Mojave $\left(54000 \mathrm{~km}^{2}\right)$ y sonorense $\left(275000 \mathrm{~km}^{2}\right)$, se tienen registradas, respectivamente, 152, 220, 257 y 272 especies (Behle, 1976; Arizmendi y Espinosa de los Monteros, 1996; Russell y Monson, 1998; Garza et al., 2004, 2007; Arizmendi y Valiente-Banuet, 2007). En la RBBM están registradas 271 especies en $960 \mathrm{~km}^{2}$. Esta diferencia puede ser debida a que la RBBM está localizada entre los climas templados y tropicales de México, y en la confluencia de las zonas neártica y neotropical. Esta situación permitiría que la RBBM tenga especies de aves de los 2 grupos de clima y de las 2 zonas biogeográficas. Esta ubicación geográfica sólo es comparable con la de la Reserva de la Biosfera Tehuacán-Cuicatlán, donde también se presentan zonas templadas. Desafortunadamente, para esta última reserva no hay aún una lista completa que contemple estas zonas (CONANP, 2003; Arizmendi y Valiente-Banuet, 2007; pero existen listas parciales para las zonas templadas en o cerca de ella (Peterson et al., 2003; Grosselet y Burcsu, 2005) y, considerando zonas desérticas y templadas, es imposible comparar su comunidad de aves con la de la RBBM. Sin embargo, para la Reserva de la Biosfera de la Michilía (Garza et al., 2007), un lugar ubicado mucho más al norte que la RBBM, y que presenta también zonas desérticas y bosques templados, se tiene una riqueza más parecida.

A pesar de que la diversidad avifaunistica en la RBBM es comparativamente alta en relación con otros sitios similares, aún falta analizar la presencia de muchas especies. Por ejemplo, en todos los trabajos hechos en la zona (Villada, 1865; Sánchez, 1978; Mancilla, 1988; el actual), no se ha estudiado a detalle la avifauna de hábitos nocturnos (se visitó sistemáticamente la vegetación de tipo templado, pero sólo se tomaron registros dispersos en otros tipos de vegetación). Faltan más trabajos en esta área para determinar con seguridad la presencia de algunas especies, ya que como se ha demostrado en otros estudios (Ortiz-Pulido y Díaz, 2001; Peterson et al., 2003; Gómez de Silva, en prensa) la extrapolación desde trabajos con enfoque nacional o internacional (Del Hoyo et al., 1992; Howell y Webb, 1995; Johnsgard, 1997), aunque valiosa, tiene limitaciones con grupos de aves, o sitios, poco estudiados. Por otra parte, falta verificar la presencia de otras especies previamente registradas, sobre todo aquellas para las que no existe evidencia confiable sobre su presencia. Este es el caso de las registradas una sola vez, por un solo autor (i.e., Villada, 1865; Sánchez, 1978; Mancilla, 1988), en los últimos 140 años: Anhinga 


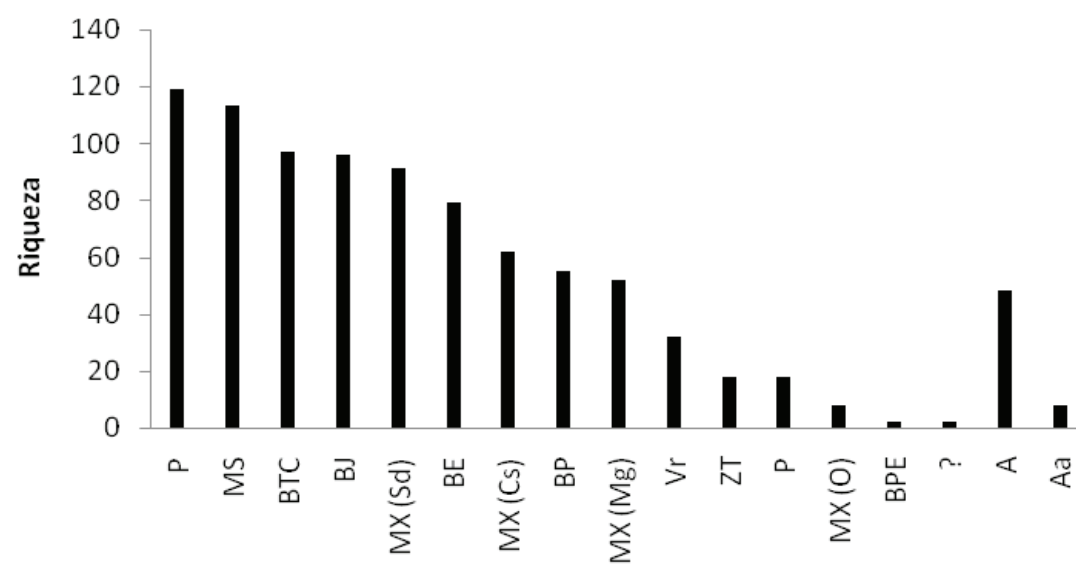

Tipos de vegetación o hábitos
Figura 2. Riqueza de especies de aves por tipo de vegetación en la Reserva de la Biosfera Barranca de Metztitlán. P, zona perturbada de La Vega; MS matorral submontano; BTC, bosque tropical caducifolio; BJ, bosque de táscate; MX (Sd), matorral xerófito con dominancia de S. dumortieri; BE, bosque de encino; MX (Cs), Matorral xerófito con dominancia de C. senilis; BP, bosque de pino; MX $(\mathrm{Mg})$, matorral xerófito con dominancia de M. geometrizans; $\mathrm{Vr}$, vegetación ribereña; $\mathrm{ZT}$, zona de transición entre bosque de tascate-matorral crasicaulematorral submontano; $\mathrm{P}$ pastizal; $\mathrm{MX}$ (O), matorral xerófito con dominancia de Opuntia sp.; BPE, bosque de pino-encino, y ?, no determinado. Se indican además especies que no entran en las categorías previas pero sí en las de hábitos acuáticos, A y aéreos, Aa.

Figura 3. Tipos de vegetación utilizados por las especies de aves en la Reserva de la Biosfera Barranca de Metztitlán.

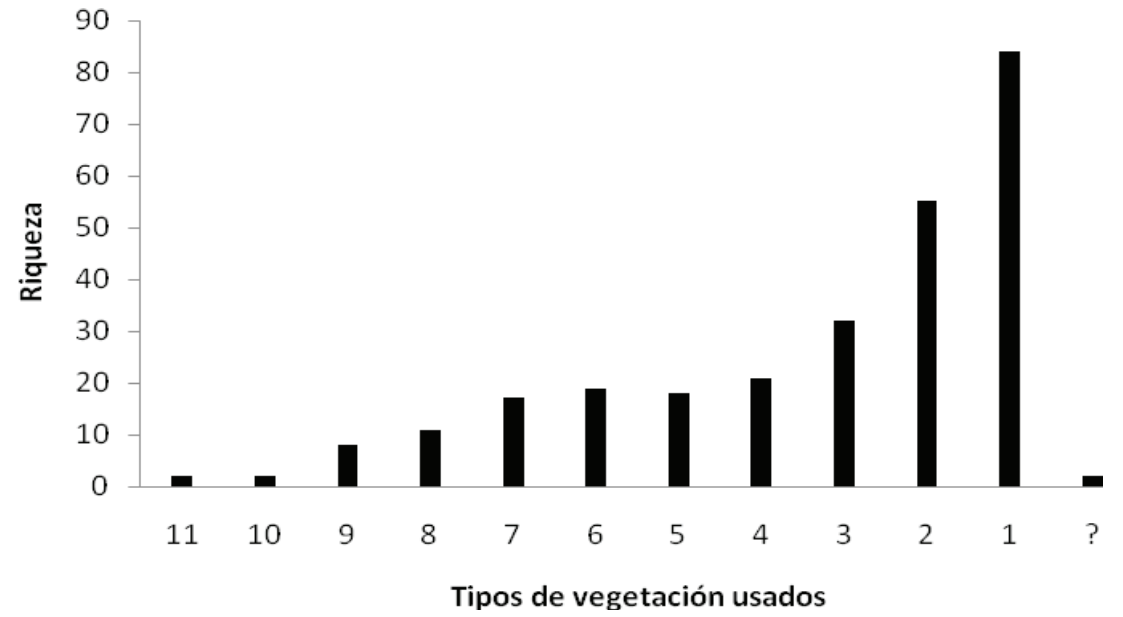

anhinga, Campephilus guatemalensis, Megarhynchus pitangua, Melospiza melodia, (especies cuya distribución está cerca de la RBBM, sensu Howell y Webb, 1995), Melanerpes hypopolius y Cacicus melanicterus (especies cuya distribución está muy lejos de la RBBM, sensu Howell y Webb 1995). Otro caso lo constituye el registro de Villada (1865) de Turdus coeruleus, una especie que fue dividida posteriormente en varias del género Sialia (Cuevas 2002). Creemos que el registro de Villada (1865) se refiere en realidad a $S$. mexicana o $S$. sialis, ya que la distribución actual de ambas abarca el área ocupada por la RBBM (sensu Howell y Webb, 1995); de hecho, Mancilla (1988) registra $S$. sialis y $S$. mexicana en zonas adyacentes a la reserva, por lo que no hay duda de que puedan estar presentes en la RBBM. Si todas estas especies fueran confirmadas para la reserva, la riqueza se incrementaría infuscatus, Anthus spinoletta, Parula americana, Wilsonia canadensis, Ergaticus ruber, Basileuterus belli, Chlorospingus ophthalmicus, Aimophila cassini y Loxia curvirostra) presentes en 2 áreas adyacentes a la RBBM (Zacualtipán y Zoquizoquipan). Muchas pueden estar ocupando zonas más tropicales, como el corredor costero del golfo de México, y no es claro si están dentro de los terrenos de la reserva, ya que, como fue notado por Sánchez (1978), muchas de las especies localizadas en este corredor parecen no invadir la zona de la Barranca de Metztitlán. Lo anterior da evidencia de la necesidad de realizar estudios sobre el tema.

Aunque el estudio de la avifauna presente en la RBBM fue iniciado siglo y medio atrás, las actividades de investigación sobre este grupo animal han sido, hasta ahora, pocas y no sistemáticas. Como es natural, confiamos 


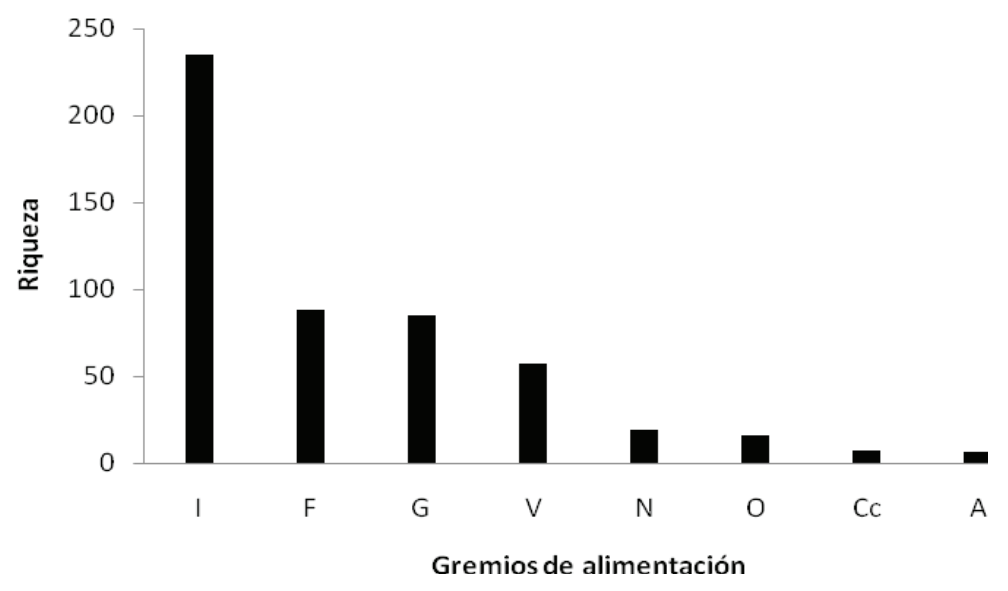

Figura 4. Número de especies de aves por gremio de alimentación en la Reserva de la Biosfera Barranca de Metztitlán. I, insectívoros; F, frugívoros; G, granívoros; V, vertebrados; $\mathrm{N}$, nectarívoros; O, omnívoros; $\mathrm{C}$, carroñeros, y A, vegetación acuática.

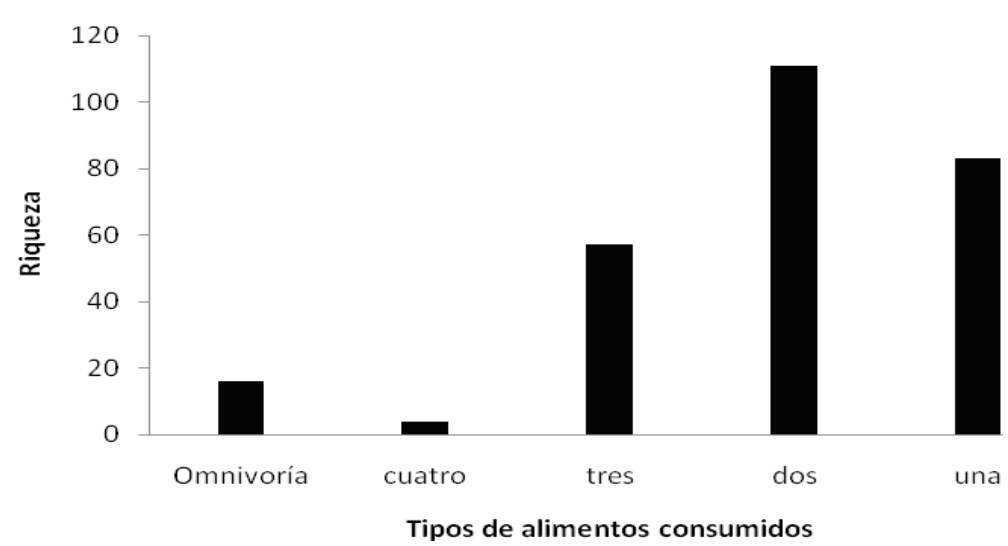

Figura 5. Número de categorías de alimento consumidas por las aves en la Reserva de la Biosfera Barranca de Metztitlán.

\section{Agradecimientos}

A la Dirección de la RBBM, especialmente a la Ing. Alejandra Rodríguez Gómez y al Ing. Salvador Montes Quintero, quienes ofrecieron muchas facilidades para realizar este trabajo. A Jorge y Alejandro, brigadistas de la RBBM, por su apoyo logístico en las salidas de campo. A Guillermo Gil y Garbó e Irving Garnica por su auxilio en el trabajo en campo. A

que en el futuro se añadirán otras especies al listado que se Miguel Ángel Martínez-Morales, Román Díaz-Valenzuela y a los revisores anónimos, por sus amables comentarios a una versión previa de este escrito. A Guillermo Gil y Garbo y Helga Caballero, por el auxilio técnico para la elaboración de la base de datos de las aves de la zona. CONACYT-SEMARNAT (proyecto 2002-CO1-0248), FOMIX-CONACyT (claves 43761 y 2008-95828), CONABIO (proyecto ASO10), SEP (a Raúl Ortiz Pulido, a través de su programa PROMEP en la UAEH), Mabio, A.C. y CONANP (a través del Programa Nacional de Monitoreo de Aves) apoyaron financieramente el trabajo de campo que hizo posible obtener los datos registrados aquí. Una beca de año sabático otorgada por CONACyT a Raúl Ortiz Pulido permitió escribir este documento.

\section{Literatura citada} estudiadas en la RBBM por su lejania, podrín reservar sorpresas avifaunísticas en el futuro, tal vez planteando la necesidad de extender los terrenos de la reserva con la intención de protegerlas.

Anderson, B., R. Homart y J. Rice. 1983. Avian and vegetation community structure and their seasonal relationships in the lower Colorado River Valley. Condor 85:392-405. 
AOU (American Ornithologists' Union). 1998. Check-list of North American birds, séptima edición. American Ornithologists' Union, Washington, D.C. 829 p.

AOU (American Ornithologists' Union). 2000. Forty-second supplement to the American Ornithologists' Union checklist of North American birds. Auk 117:847-858.

Arellano, M. y P. Rojas. 1956. Aves acuáticas migratorias en México. I. Instituto Mexicano de Recursos Naturales Renovables, México, D.F. 270 p.

Arizmendi, M. C. y A. Espinoza de los Monteros. 1996. Avifauna de los bosques de cactáceas columnares del valle de Tehuacán, Puebla. Acta Zoológica Mexicana (nueva serie) 67:25-46.

Arizmendi, M. C. y L. Valdelamar (eds.). 1999. Áreas de Importancia para la conservación de las aves en México. CIPAMEX, México, D.F. 440 p.

Arizmendi, M. C. y A. Valiente-Banuet. 2007. Aves de la Reserva de la Biosfera Tehuacán Cuicatlán. UNAM / CONABIO / Fundación Cuicatlán, México, D.F. 164 p.

Arregui, L. 2004. ¿A qué nivel de la escala espacial seleccionan los colibríes su área de forrajeo? Diploma de estudios avanzados. Universidad Autónoma de Madrid. 27 p.

Banks, R. C., C. Cicero, J. L. Dunn, A. W. Kratter, P. C. Rasmussen, J. V. Remsen, J. D. Rising y D. F. Stotz. 20022007. Forty-third - Forty-seventh supplement to the American Ornithologists' Union check-list of North American birds. Auk 119:897-906; 120:923-931; 121:985-995; 122:10261031; 123:926-936.

Banks, R. C., Chesser, R. T., C. Cicero, J. L. Dunn, A. W. Kratter, I. J. Lovette, P. C. Rasmussen, J. V. Remsen, J. D. Rising y D. F. Stotz. 2007. Forty-eighth supplement to the American Ornithologists' Union check-list of North American birds. Auk 124:1109-1115.

Banks, R. C., Chesser, R. T., C. Cicero, J. L. Dunn, A. W. Kratter, I. J. Lovette, P. C. Rasmussen, J. V. Remsen, J. D. Rising, D. F. Stotz y K. Winker. 2008. Forty-eighth supplement to the American Ornithologists' Union check-list of North American birds. Auk 125:758-768.

Beedy, E.1981. Bird communities and forest structure in the Sierra Nevada of California. Condor 83:97-105.

Begon, M., Townsend, C. R. y J. L. Harper. 2006. Ecology, from individuals to ecosystems. Blackwell, Malden, Massachusetts. 738 p.

Behle, W. H. 1976. Mohave desert avifauna in the Virgin River Valley of Utah, Nevada, and Arizona. Condor 78:40-48.

Bibby, C. J., N. D. Burgess, D. A. Hill y S. Mustoe. 2000. Bird census techniques, segunda edición. Academic, London. $350 \mathrm{p}$.

Bjelland, A. D. y J. C. Ray. 1977. Birds collected in the state of Hidalgo, México. Ocassional Papers Museum Texas Technological University 46:1-32.

Cantú, S. 1953. La vega de Metztitlán en el estado de Hidalgo. Boletín de la Sociedad Mexicana de Geografía y Estadística $75: 1-3$.

CONANP (Comisión Nacional de Áreas Naturales Protegidas). 2003. Programa de Manejo de la Reserva de la Biosfera Barranca de Metztitlán. Comisión Nacional de Áreas Naturales Protegidas, SEMARNAT, México,
D.F. 204 p.

Cueto, V. y J. López. 1999. Determinants of bird species richness: role of climate and vegetation structure at a regional scale. Journal of Biogeography 26:487-492.

Cuevas, M. C. 2002. Un científico mexicano y su sociedad en el siglo XIX. Universidad Autónoma del Estado de Hidalgo/ Sociedad Mexicana de Historia de la Ciencia y la Tecnología, Pachuca, Hidalgo. 240 p.

Del Hoyo, J., A. Elliot y J. Sargatal (eds.). 1992. Handbook of the birds of the World, vol. 1, Ostrich to Ducks. Birdlife International y Lynx, Barcelona. $696 \mathrm{p}$.

Del Hoyo, J., A. Elliot y J. Sargatal (eds.). 1996. Handbook of the birds of the world, vol. 3, Hoatzin to Auks. Birdlife International y Lynx, Barcelona. $821 \mathrm{p}$.

Del Hoyo, J., A. Elliot y J. Sargatal (eds.). 1999. Handbook of the birds of the world, vol. 5, Barn-owls to Hummingbirds. Birdlife International y Lynx, Barcelona. 759 p.

Del Hoyo, J., A. Elliot y J. Sargatal (eds). 2005. Handbook of the birds of the world, vol. 10, Cuckoo-shrikes to Thrushes. Birdlife International y Lynx, Barcelona. 895 p.

Díaz-Valenzuela, R. 2008. Análisis descriptivo del sistema colibrí-planta en tres niveles de las escalas espacial, temporal y en la jerarquía ecológica en un paisaje mexicano. Diploma de estudios avanzados. Centro Iberoamericano de la Biodiversidad / Instituto de Ecología / Universidad Autónoma del Estado de Hidalgo, Alicante. 60 p.

DGFS (Dirección General de Fauna Silvestre). 1980. Informe técnico del programa "Desarrollo faunístico del estado de Hidalgo". Departamento de Planeación y Proyectos, DGFS, México D.F. (sin foliar).

Friedman, H., L. Griscom y R. T. Moore. 1950. Distributional check-list of the birds of México. Part I. Pacific Coast Avifauna 29, Cooper Ornithological Society, Berkeley, California. 202 p.

Garza, A., M. Neri y E. E. Arangón. 2004. Guía de aves Reserva de La Michilía. INECOL / CONABIO, Xalapa, Veracruz, México. 184 p.

Garza, A., E. E. Aragón, M. Neri, A. Sánchez, G. D. de León y S.R. Gutíerrez. 2007. Guía de aves de la Reserva de la Biosfera de Mapimí. Instituto de Ecología, AC, Fondos Mixtos de Fomento a la Investigación Científica y Tecnológica y Centro de Recursos para el Análisis de Conflictos; Durango, Durango. 179 p.

Gómez de Silva, H. En prensa. Aves en México: registros estatales recientes. In Avifaunas estatales de México, R. Ortiz-Pulido, A. Navarro-Sigüienza, H. Gómez de Silva, O. Rojas y T. Peterson (eds.). Sociedad para el Estudio y Conservación de las Aves en México A.C. Pachuca, Hidalgo.

González-García, F. y H. Gómez de Silva. 2003. Especies endémicas: patrones de distribución y retos para su conservación. In Conservación de aves, experiencias en México, H. Gómez de Silva y A. Olivares de Ita (eds.). NFWF, CIPAMEX y CONABIO, México, D.F., México. p. 150-194.

Grosselet, M. y T. Burcsu. 2005. Notas sobre las aves de Calpulalpan de Méndez, Sierra Juárez, Oaxaca, México. Huitzil 6:18-24.

Hernández-Avilés, J., A. Guzmán S. y M. C. López G. 2003. Aves 
de la Barranca de Metztitlán. In Metzttilán: lugar de la luna y las maravillas, M. A. Armella, M. L. Yañez y E. Sandoval (Coordinadores). Universidad Autonoma Metropolitana. México, D.F. p. 43-52.

Howell, S. N. G. 2002. Hummingbirds of North America. The Photographic Guide. Princeton University Press, Princeton, New Jersey. 272 p.

Howell, S. N. G. y S. Webb. 1995. A guide to the birds of Mexico and Northern Central America. Oxford University Press, Oxford. $851 \mathrm{p}$.

Johnsgard, P. A. 1997. The Hummingbirds of North America, segunda edición. Smithsonian Institution Press, Washington, D.C. 314 p.

Mancilla, M. 1988. Estudio preliminar de la avifauna en el transecto Zacualtipan-Zoquizoquipan-San Juan Metztitlán en el este de Hidalgo, México. Tesis, Facultad de Ciencias, Universidad Autónoma de México, México, D.F. 85 p.

Martín del Campo, R. 1936. Contribución al conocimiento de la fauna de Actopan, Hidalgo. IV. Vertebrados observados en la época de secas. Anales del Instituto de Biología, Universidad Nacional Autónoma de México 7:271-286.

Martín del Campo, R. 1937. Notas acerca de las aves y los mamíferos del Valle de Metztitlán, Hidalgo. Anales del Instituto de Biología, Universidad Nacional Autónoma de México 8:268-272.

Martínez-García, V. 2006. Interacciones colibrí-planta en tres tipos de vegetación de la Reserva de la Biosfera Barranca de Metztitlan, Hidalgo, México. Tesis, Laboratorio de Ecología de Poblaciones, Universidad Autónoma del Estado de Hidalgo, Pachuca. 57 p.

Martínez-Morales, M. A. 2004. Nuevos registros de aves en el bosque mesófílo de montaña del noreste de Hidalgo, México. Huitzil 5:12-19.

Martínez-Morales, M. A., R. Ortiz-Pulido, B. de la Barreda, I. L. Zuria, J. Bravo-Cadena y J. Valencia-Herverth. 2007. Hidalgo. In Avifaunas estatales de México, R. OrtizPulido, A. Navarro-Sigüenza, H. Gómez de Silva, O. Rojas-Soto y T. A. Peterson (eds.). CIPAMEX, Pachuca, Hidalgo. p. 49-95.

Mauricio-López E. 2005. Interacción colibrí-planta: variación espacial en un matorral xerófilo de Hidalgo, México. Tesis, Universidad Autónoma del Estado de Hidalgo, Pachuca. 8 p.

Miller, A., H. Griscom, y R. T. Moore, R. T. 1957. Distributional check-list of the birds of Mexico. Part II, Pacific Coast Avifauna 33. Cooper Ornithological Society, Berkeley, California. $436 \mathrm{p}$.

National Geographic Society. 2002. Field guide to the birds of North America, cuarta edición. National Geographic Society, Washington, D. C. 480 p.

Navarro-Siguienza, A.G. y A.T. Peterson. 2004. An alternative species taxonomy of the birds of Mexico. Biota Neotropica 4:1-32.

Ortiz-Pulido,, R., H. Gómez de Silva, F. González-García y A. Álvarez. 1995. Avifauna del Centro de Investigaciones Costeras La Mancha, Veracruz, México. Acta Zoológica Mexicana (nueva serie) 66:87-118.

Ortiz-Pulido, R. y R. Díaz. 2001. Distribución y densidad de colibríes en la zona baja del centro de Veracruz, México. Ornitología Neotropical 12:297-317.

Ortiz-Pulido, R. y V. Martínez-García. 2006. A female Lucifer Hummingbird (Calothorax lucifer) with iridescent chin feathers. Journal of Field Ornithology 77:71-73.

Ortiz-Pulido, R., V. Martínez-García y J. Bravo-Cadena. 2006. Colibríes, Reserva de la Biosfera Barranca de Metztitlán. Universidad Autónoma del Estado de Hidalgo, Pachuca, Hidalgo. 16 p.

Ortiz-Pulido, R., E. Mauricio-López, V. Martínez-García y J. Bravo-Cadena. 2008. ¿Sabes quién vive en el Parque Nacional El Chico? Colibríes. Universidad Autónoma del Estado de Hidalgo/Secretaría de Educación Pública Hidalgo/Dirección del Parque Nacional El Chico. Pachuca, Hidalgo. 22 p.

Peterson, A. T., G. Escalona-Segura, K. Zyskowski, D. A. Kluza y B. E. Hernández-Baños. 2003. Avifaunas of two dry forests sites in northern Oaxaca, Mexico. Huitzil 4:3-9.

Peterson, R. T. y E. L. Chalif. 1989. Aves de México. Diana, México, D.F. 473 p.

Pichardo, J. 1987. Estudio ornitológico en el Municipio de Alfajayucan y áreas adyacentes, estado de Hidalgo. Tesis, Escuela Nacional de Estudios Profesionales, Iztacala, Universidad Nacional Autónoma de México, México, D.F. $115 \mathrm{p}$.

Ramírez, B. P., A. E. Desucre-Medrano, A. G.Navarro S., P. J. Romo O. y H. J. Castro. 1994. Winter specimen of the Common Loon (Gavia immer) from the state of Hidalgo, México. Southwestern Naturalist 39:394-395.

Rojas-Soto, O., L. A. Sánchez-González y S. López de Aquino. 2002. New information on birds of northern Hidalgo, Mexico. Southwestern Naturalist 47:471-475.

Rzedowski, J. 2005. Vegetación de México, edición digital, CONABIO, México, D.F. 504 p. www.conabio.gob.mx/ institucion/centrodoc/doctos/vegetacion_de_mexico.html. Última consulta: 26.IV.2009.

Russell, S. M. y G. Monson. 1998. The birds of Sonora. University of Arizona Press, Tucson. 360 p.

Sánchez, H. 1978. Manual de campo de cactáceas y suculentas de la Barranca de Metztitlán, Hidalgo. Sociedad Mexicana de Cactología, Publicación de Difusión Cultural 2:1-86.

SEMARNAT (Secretaría de Medio Ambiente y Recursos Naturales). 2002. Norma Oficial Mexicana NOM-059ECOL-2001. Proteccion ambiental- Especies nativas de México de flora y fauna silvestres- Categorías de riesgo y especificaciones para su inclusión, exclusión o cambio Lista de especies en riesgo. Diario Oficial de la Federación, segunda sección, 6 de marzo, 1-81.

Tokeshi, M. 1993. Species abundance patterns and community structure. Advances in Ecological Research 24:112-186.

Valencia-Herverth, J., R. Valencia-Herverth y F. MendozaQuijano. 2008. Registros adicionales de aves para Hidalgo, México. Acta Zoológica Mexicana (nueva serie) 24:115123.

Valencia-Herverth, R., J. Valencia-Herverth y F. MendozaQuijano. 2009. Corvus imparatus, primer registro para Hidalgo, México. Huitzil 10:15-18.

Villada, M. 1865. Estudios sobre la fauna de Pachuca, Real del 
Monte, Mineral del Chico y Barranca Honda. Memoria de los trabajos ejecutados por la Comisión Científica de Pachuca en el año de 1864, R. Almaraz (ed.). Edición facsimilar. 1993. V. Ballesteros (comp.), Universidad Autónoma del Estado de Hidalgo, Pachuca. 358 p.

Villavicencio, M. A., Y. Marmolejo y B. E. Pérez. 1993. Investigaciones recientes sobre flora y fauna de Hidalgo,
México. Universidad Autónoma de Hidalgo, Pachuca, Hidalgo, México. 515 p.

Whitmore, R. 1975. Habitat ordination of passerine birds of the Virgin River Valley, Southwestern Utah. Wilson Bulletin 87:65-74.

Williamson, S. L. 2001. Hummingbirds of North America, Peterson Field Guides. Houghton Mifflin, New York. 263 p.

Apéndice 1. Especies de aves presentes en la Reserva de la Biosfera Barranca de Metztitlán, Hidalgo; México. Se indica estacionalidad; abundancia relativa; tipo de vegetación usado; alimentación; estatus de conservación en México y endemismo. Estacionalidad: M, migratoria; R, residente; O, ocasional, y ?, sin datos para determinarla en la zona. Abundancia relativa: $\mathbf{R}$, rara; $\mathbf{C}$, común; A, abundante, y ?, no determinada. Tipos de vegetación o hábitos: BTC, bosque tropical caducifolio; MX (Mg), matorral xerófito con dominancia de Myrtillocactus geometrizans; MX (Sd), matorral xerófito con dominancia de Stenocereus dumortieri; MX (Cs), matorral xerófito con dominancia de Cephalocereus senilis; MX (O), matorral xerófito con dominancia de Opuntia sp.; MS, matorral submontano; BJ, bosque de tascate; BP, bosque de pino; BE, bosque de encino; BPE, bosque de pino-encino; ZT, zona de transición entre bosque de táscate-matorral xerófito-matorral submontano; PA, pastizal; P, zona perturbada de La Vega; Vr, vegetación ribereña; A, hábitos acuáticos; Ae, hábitos aéreos, y ?, no determinado. Alimentación: Cc, carroñero; G, granívoro; F, frugívoro; V, vertebrados; I, insectívoros; N,nectarívoro; A, vegetación acuática, y O, omnívoro. Estatus de protección: A, amenazada, y Pr, protección especial. Endemismo: E, endémica de México; CE, cuasiendémicas y SE, semiendémicas. Especies registradas por: aSánchez (1978), bancilla (1988), ${ }^{\mathrm{c}}$ Villada (1865).

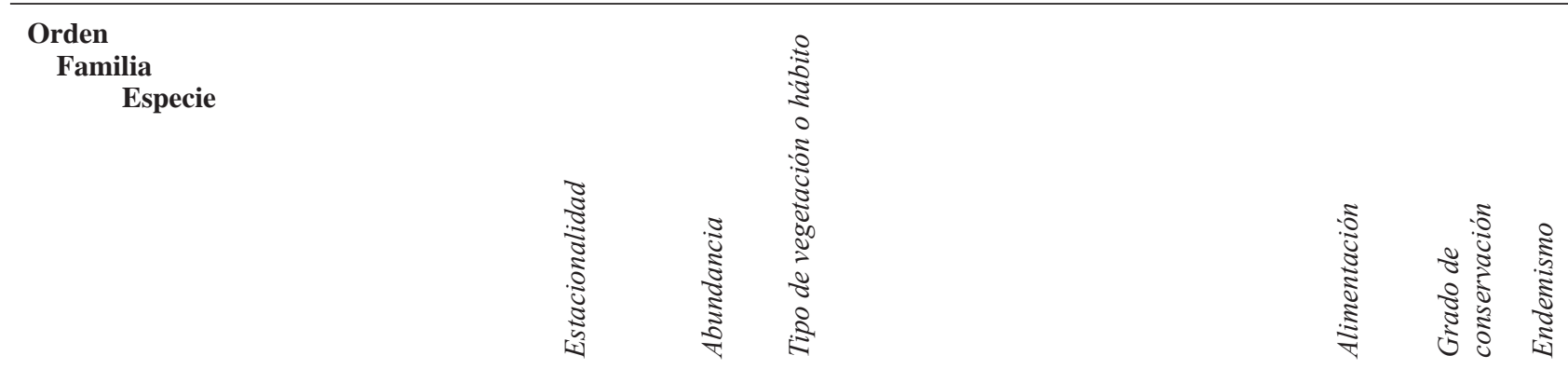

\section{Anseriformes \\ Anatidae}

Aix sponsa

Anas strepera

Anas platyrhynchos

Anas discors

Anas cyanoptera

Anas clypeata

Anas acuto

Anas crecca

Aythya valisineria

Aythya collaris

Oxyura jamaicensis

\section{Galliformes}

Odontophoridae

Callipepla squamata

Dactylortyx thoracicus

\section{Podicipediformes}

Podicipedidae

Tachybaptus dominicus

$\begin{array}{ccc}\mathrm{M} & ? & \mathrm{~A} \\ \mathrm{M} & \mathrm{R} & \mathrm{A} \\ \mathrm{R}, \mathrm{M} & \mathrm{C} & \mathrm{A} \\ \mathrm{M} & \mathrm{R} & \mathrm{A} \\ \mathrm{M} & \mathrm{R} & \mathrm{A} \\ \mathrm{M} & \mathrm{C} & \mathrm{A} \\ \mathrm{M} & \mathrm{C} & \mathrm{A} \\ \mathrm{M} & \mathrm{C} & \mathrm{A} \\ \mathrm{M} & ? & \mathrm{~A} \\ \mathrm{M} & ? & \mathrm{~A} \\ \mathrm{R} & \mathrm{R} & \mathrm{A}\end{array}$

A

$\mathrm{O}$

$\mathrm{O}$

A

IA

$\mathrm{O}$

IGA

GI

GI

AIV

AI

IG

GIF

GI Pr E

$\begin{array}{llllll}\mathrm{R} & \mathrm{R} & \mathrm{MX}(\mathrm{Sd}), \mathrm{Ms} & & & \\ \mathrm{R} & \mathrm{BPE} & \text { GI } & \text { Pr } & \mathrm{E}\end{array}$

M $\quad$ R A

IV Pr 
$\begin{array}{ll} & \text { Podilymbus podiceps } \\ \text { a, c } & \text { Aechmophorus occidentalis }\end{array}$

Pelecaniformes

\section{Pelecanidae}

Pelecanus erythrorhynchos

Pelecanus occidentalis

Phalacrocoracidae

Phalacrocorax brasilianus

\section{Ciconiiformes}

\section{Ardeidae}

Ardea herodias

Ardea alba

Egretta thula

Egretta caerulea

Egretta tricolor

Bubulcus ibis

Butorides virescens

Nycticorax nycticorax

Threskiornithidae

Eudocimus albus

Plegadis chihi

\section{Falconiformes}

Cathartidae

Coragyps atratus

Cathartes aura

\section{Accipitridae}

Pandion haliaetus

Elanus leucurus

Circus cyaneus

Accipiter striatus

Accipiter cooperi

Buteo nitidus

Buteo swainsoni

Buteo jamaicensis

\section{Falconidae}

Caracara cheriway

Falco sparverius

Falco columbarius

Falco peregrinus

\section{Gruiformes}

\section{Rallidae}

a Porzana carolina Gallinula chloropus

Fulica americana

$\begin{array}{lll}\mathrm{M} & \mathrm{R} & \mathrm{A} \\ \mathrm{R} & ? & \mathrm{~A}, \mathrm{P}\end{array}$

VI

V

M A A

IV

M $\quad$ R A

V

M $\quad$ C $\quad$ A

V

$\begin{array}{llll}\mathrm{R}, \mathrm{M} & \mathrm{R} & \mathrm{A}, \mathrm{P} & \mathrm{IV}\end{array}$

R, M C A, P IV

R, M C A, P IV

M C A

M R A

R A P

M C A

R C A, P IV

$\begin{array}{lllll}M & \text { R } & \text { A } & \text { IV }\end{array}$

R, M C A IV

R, M R BTC, MX (Cs, Mg y Sd), MS, BJ, P Cc

R C BTC, MX (Cs, Mg y Sd), MS, P, BJ, BE Cc

M R A $\quad$ V

R $\quad \mathrm{R} \quad \mathrm{MS}, \mathrm{BJ} \quad \mathrm{IV}$

M R MX (Sd), P, PA

$\begin{array}{lllll}\mathrm{R} & \mathrm{R} & \mathrm{BTC}, \mathrm{MS}, \mathrm{MX}(\mathrm{Sd}) & \mathrm{CcV} & \mathrm{Pr}\end{array}$

$\begin{array}{lllll}\mathrm{R} & \mathrm{R} & \mathrm{BTC}, \mathrm{MX}(\mathrm{Mg} \text { y Sd}), \mathrm{MS}, \mathrm{P}, \mathrm{BJ}, \mathrm{VR} & \mathrm{CcV} & \mathrm{Pr}\end{array}$

? $\quad \mathrm{R} \quad \mathrm{P} \quad$ IV

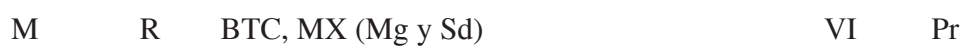

R R BTC, MX (Cs, Mg y Sd), MS, BE, P, BJ, V PA, ZT

R $\quad \mathrm{C} \quad \mathrm{BJ}, \mathrm{BE}, \mathrm{P}, \mathrm{MX}(\mathrm{Cs}) \quad \mathrm{CcV}$

M R MX (Mg y Sd), MS, BE, BTC, P, PA ICc

M $\quad \mathrm{R} \quad \mathrm{MX}(\mathrm{Sd}), \mathrm{P} \quad \mathrm{VI}$

$\begin{array}{lllll}\mathrm{R} & \mathrm{R} & \mathrm{P}, \mathrm{BTC}, \mathrm{MX}(\mathrm{O}, \mathrm{Sd}) & \mathrm{V} & \mathrm{Pr}\end{array}$

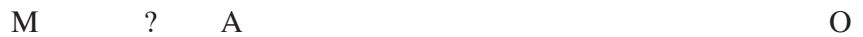

$\begin{array}{llll}\mathrm{R} & \mathrm{A} & \mathrm{A}, \mathrm{P} & \mathrm{GVI}\end{array}$

R A A, P GVI 


\section{Gruidae}

Grus canadensis

$\mathrm{M} \quad$ ? $\mathrm{P}$

$\mathrm{O} \quad \mathrm{Pr}$

\section{Charadriiformes}

\section{Charadriidae}

Charadrius vociferus

R $\quad \mathrm{R} \quad \mathrm{A}, \mathrm{PA}$

\section{Recurvirostridae}

Recurvirostra americana

M $\quad$ R $A$

IG

Scolopacidae

Actitis macularius

Tringa melanoleuca

Tringa flavipes

Limosa haemastica

Calidris minutilla

Calidris mauri

Calidris bairdii

Limnodromus scolopaceus

Gallinago delicata

$\begin{array}{ccc}\mathrm{O} & \mathrm{R} & \mathrm{A}, \\ \mathrm{M} & \mathrm{R} & \mathrm{A} \\ \mathrm{M} & \mathrm{R} & \mathrm{A} \\ ? & ? & \mathrm{~A} \\ \mathrm{M} & \mathrm{R} & \mathrm{A} \\ \mathrm{M} & \mathrm{R} & \mathrm{A} \\ \mathrm{M} & ? & \mathrm{~A} \\ \mathrm{M} & ? & \mathrm{~A} \\ \mathrm{M} & ? & \mathrm{~A},\end{array}$

IG

IV

IV

IG

I

IG

I

IGA

Laridae

Leucophaeus pipixcan

R A

Larus delawarensis

M $\quad$ C $A$

IV

\section{Columbiformes}

\section{Columbidae}

Columba livia

Patagioenas fasciata

Streptopelia decaocto

Zenaida asiatica

Zenaida macroura

Columbina inca

Columbina passerina

Columbina talpacoti

Leptotila verreauxi

Cuculiformes

\section{Cuculidae}

Piaya cayana

Coccyzus americanus

Geococcyx californianus

Crotophaga sulcirostris

\section{Stringiformes}

Stringidae

Otus flammeolus

Megascops trichopsis

Bubo virginianus

c Glauciduim gnoma 
Glaucidium brasilianum

Micrathene whitneyi

Athene cunicularia

Asio flammeus

Aegolius acadicus

\section{Caprimulgiformes}

\section{Caprimulgidae}

Chordeiles acutipennis

Apodiformes

\section{Apodidae}

Cypseloides niger

Aeronautes saxatalis

Trochilidae

Cynanthus latirostris

Hylocharis leucotis

Amazilia violiceps

Lampornis clemenciae

Eugenes fulgens

Calothorax lucifer

Archilochus colubris

Archilochus alexandri

Stellula calliope

Selasphorus platycercus

Selasphorus rufus

Selasphorus sasin

Trogoniformes

Trogonidae

Trogon mexicanus

\section{Coraciiformes}

\section{Alcedinidae}

Megaceryle torquatus

Megaceryle alcyon

Chloroceryle americana

\section{Piciformes}

Picidae

Melanerpes formicivorus Melanerpes aurifrons

Sphyrapicus varius

Picoides scalaris

Picoides villosus

Colaptes rubiginosus

Colaptes auratus

$\begin{array}{ccl}\mathrm{R} & ? & \mathrm{VR} \\ ? & ? & \mathrm{BE}, \mathrm{P}, \mathrm{BJ} \\ \mathrm{M} & \mathrm{R} & \mathrm{MS} \\ ? & ? & \mathrm{BE}, \mathrm{P} \\ ? & ? & \mathrm{BE}\end{array}$

IV

IV

IV

V

IV

Pr SE

SE
$\mathrm{R} \quad \mathrm{R} \quad \mathrm{BTC}, \mathrm{MC}(\mathrm{Cs}, \mathrm{Sd}) \quad \mathrm{I}$

$\begin{array}{llll}\mathrm{R} & \mathrm{R} & \mathrm{MX}(\mathrm{Mg}), \mathrm{MS} & \mathrm{I} \\ \mathrm{R} & \mathrm{R} & \mathrm{BP}, \mathrm{BE}, \mathrm{P} & \mathrm{I}\end{array}$

R A BTC, MX (Cs, Mg y Sd), MS, P, BJ, ZT NI NE

$\mathrm{R} \quad \mathrm{A} \quad \mathrm{BTC}, \mathrm{MX}(\mathrm{Mg}$ y Sd), MS, BP, BE, P, BJ NI

O $\quad \mathrm{R} \quad \mathrm{BTC}, \mathrm{MS}, \mathrm{P}, \mathrm{VR} \quad \mathrm{NI}$ SE

R C BTC, MX (Mg y Sd), MS, BE, P, BJ NIF SE

$\begin{array}{llll}\mathrm{R} & \mathrm{C} & \mathrm{MX}(\mathrm{Mg} \text { y Sd), MS, BP, BE, P, BJ NI }\end{array}$

R C BTC, MX (Cs, Mg, O y Sd), MS, BP, BE, NI SE

$\begin{array}{llll} & \text { BJ, ZT, P } \\ \text { O } & \text { MS, MX (Sd), BJ, BTC NI }\end{array}$

O R MS, BTC, MX (Sd) NI

M $\quad \mathrm{R} \quad \mathrm{MX}(\mathrm{Sd}), \mathrm{BE}, \mathrm{P} \quad \mathrm{NI} \quad$ SE

R R MS, BJ, BTC, MX (Sd) NI

? $\mathrm{R} \quad \mathrm{BJ} \quad \mathrm{NI}$

O R MX (Sd) NI

SE

Passeriformes 


\section{Furnariidae}

Lepidocolaptes affinis

\section{Tyrannidae}

Camptostoma imberbe

Mitrephanes phaeocercus

Contopus pertinax

Contopus cooperi

Contopus sordidulus

Contopus virens

Empidonax virescens

Empidonax minimus

Empidonax wrightii

Empidonax oberholseri

Empidonax fulvifrons

Sayornis nigricans

Sayornis phoebe

Sayornis saya

Pyrocephalus rubinus

Myiarchus cinerascens

Myiarchus nuttingi

Myiarchus tyrannulus

Pitangus sulphuratus

Miyiozetetes similis

Myiodynastes luteiventris

Tyrannus vociferans

Tyrannus verticalis

Tyrannus forficatus

Incertae sedis

Pachyramphus aglaiae

\section{Laniidae}

Lanius ludovicianus

\section{Vireonidae}

Vireo bellii

Vireo solitarius

Vireo huttoni

Vireo flavifrons

Vireo plumbeus

Vireo casinii

Vireo gilvus

Vireo olivaceus

Vireolanius melitophrys

\section{Corvidae}

Cyanocitta stelleri

\begin{tabular}{|c|c|c|c|}
\hline $\mathrm{R}$ & $\mathrm{R}$ & $\mathrm{BE}$ & IV \\
\hline $\mathrm{R}$ & $\mathrm{R}$ & MS, BTC, MX (Cs), BJ & I \\
\hline$?$ & $\mathrm{R}$ & BPE, P & I \\
\hline $\mathrm{R}$ & $\mathrm{R}$ & BTC, P, MS, MC(Cs y Sd), BJ & NIG \\
\hline M & $?$ & MS & I \\
\hline $\mathrm{R}$ & $?$ & BJ, MS, P, MX (O), BTC & IF \\
\hline M & $\mathrm{R}$ & BTC, MX (Cs, Sd y Mg), BE, MS & NIG \\
\hline M & $\mathrm{R}$ & $\mathrm{BE}, \mathrm{MX}(\mathrm{Cs}), \mathrm{BTC}$ & I \\
\hline $\mathrm{O}$ & $?$ & VR, MS, ZT & IF \\
\hline M & $\mathrm{R}$ & MS, BP, MX (Sd) & I \\
\hline M & $\mathrm{R}$ & MS, BTC, MX (Cs y Sd) & I \\
\hline $\mathrm{R}$ & $\mathrm{R}$ & $\mathrm{BP}, \mathrm{VR}, \mathrm{MS}$ & I \\
\hline $\mathrm{R}$ & $\mathrm{R}$ & $\mathrm{A}, \mathrm{P}, \mathrm{VR}, \mathrm{MX}(\mathrm{Sd}), \mathrm{ZT}$ & I \\
\hline M & $\mathrm{R}$ & MX (Mg y Sd), P, MS & IFV \\
\hline $\mathrm{R}$ & $\mathrm{R}$ & BTC, MX (Cs, Mg, O y Sd), P, MS, BJ & I \\
\hline $\mathrm{R}$ & $\mathrm{R}$ & BTC, MX (Mg, O y Sd), P, MS, BJ, ZT & IF \\
\hline $\mathrm{R}$ & A & BTC, MX (Cs, Mg y Sd), MS, BJ & IF \\
\hline$?$ & $\mathrm{C}$ & BTC, MX (Cs y Sd) & IF \\
\hline $\mathrm{O}$ & $\mathrm{R}$ & BTC, MS, MX (Cs y Sd) & IF \\
\hline $\mathrm{O}$ & $\mathrm{C}$ & BTC, P, VR & $\mathrm{F}$ \\
\hline $\mathrm{R}$ & $\mathrm{C}$ & P, BTC, MX (Cs, Sd), MS, VR & IF \\
\hline$?$ & $\mathrm{R}$ & $\mathrm{VR}, \mathrm{MX}(\mathrm{Cs})$ & I \\
\hline $\mathrm{R} ? \mathrm{M}$ ? & $\mathrm{C}$ & $\begin{array}{l}\text { BTC, MX (Cs, Mg, O y Sd), MS, P, BJ, } \\
\text { PA }\end{array}$ & IFV \\
\hline M & $\mathrm{C}$ & BTC, MX (Cs, Mg), MS, BJ, PA & IF \\
\hline$?$ & $?$ & MS, P & IF \\
\hline $\mathrm{R}$ & $\mathrm{R}$ & MS, BTC, P, VR & IF \\
\hline $\mathrm{R}$ & $\mathrm{R}$ & MX (Mg y Sd), MS, P, BJ, PA, ZT & IV \\
\hline M & $\mathrm{R}$ & BTC, MX (Mg y Sd), BP, BE & I \\
\hline M & $\mathrm{R}$ & BTC, BE, P, BJ & I \\
\hline $\mathrm{R}$ & $\mathrm{R}$ & $\mathrm{BP}, \mathrm{BJ}, \mathrm{BE}$ & I \\
\hline M & $?$ & $\mathrm{MX}(\mathrm{Sd}), \mathrm{VR}$ & IF \\
\hline M & $?$ & $\mathrm{BJ}$ & I \\
\hline M & $?$ & $\mathrm{BJ}$ & I \\
\hline M & $\mathrm{R}$ & BTC, BJ & I \\
\hline M & $\mathrm{C}$ & BTC, MX (Cs, Mg), MS, BE, BJ & IF \\
\hline$?$ & $?$ & $\mathrm{BJ}$ & IF \\
\hline
\end{tabular}

R $\quad \mathrm{R} \quad \mathrm{BP}$
SE 
Cyanocorax yncas

Aphelocoma ultramarina

Aphelocoma unicolor

Corvus corax

\section{Alaudidae}

Eremophila alpestris

\section{Hirundinidae}

Tachycineta albilinea

Tachycineta thalassina

Stelgidopteryx serripennis

Hirundo rustica

\section{Paridae}

Poecile sclateri

Baeolophus wollweberi

Baeolophus bicolor

\section{Remizidae}

Auriparus flaviceps

\section{Aegithalidae}

Psaltriparus minimus

\section{Certhiidae}

Certhia americana

Troglodytidae

Campylorhynchus gularis

Campylorhynchus

brunneicapillus
Salpinctes obsoletus

Catherpes mexicanus

Thryothorus maculipectus

Thryomanes bewickii

Troglodytes aedon

Cistothorus palustris

\section{Regulidae}

Regulus calendula

Sylviidae

Polioptila caerulea

\section{Turdidae}

c Sialia sp.

Myadestes occidentalis

Myadestes unicolor

Catharus aurantiirostris

Catharus occidentalis

Catharus mexicanus

Catharus guttatus

Turdus grayi

$\begin{array}{lll}\text { R } & \text { R } & \text { MS, VR } \\ \text { R } & \text { C } & \text { BP, BE, BJ, MS } \\ \text { O } & \text { R } & \text { BE } \\ \text { R } & \text { R } & \text { MS, P, BTC }\end{array}$

IVGF

$\mathrm{O}$

O A

$\mathrm{O}$

R $\quad$ R MS

GI

$\mathrm{R} \quad \mathrm{C} \quad \mathrm{Ae}$

R R Ae, BJ, MS I

$\mathrm{R} \quad \mathrm{C} \quad \mathrm{Ae}, \mathrm{P}, \mathrm{BJ}, \mathrm{MX}(\mathrm{Cs}) \quad$ I

R R Ae, BTC, MX (Mg), MS, P I

$\begin{array}{llll}\mathrm{O} & \mathrm{R} & \mathrm{BP} & \mathrm{IG}\end{array}$

R $\mathrm{R} \quad \mathrm{BP}, \mathrm{BE}, \mathrm{BJ} \quad \mathrm{IG}$

O R BTC, MX (Mg, Sd, Cs), P, MS, BJ, VR IG

R A BTC, MX (Cs, Mg y Sd), MS, P, BJ IG

$\begin{array}{llll}\mathrm{R} & \mathrm{C} & \mathrm{BP}, \mathrm{BE}, \mathrm{MS}, \mathrm{BJ} & \mathrm{IG}\end{array}$

R $\quad \mathrm{R} \quad \mathrm{BP} \quad \mathrm{I}$

R R BTC, MS, P, VR, ZT IV

E

R $\mathrm{R}$ MS I

R $\quad \mathrm{R} \quad \mathrm{BTC}, \mathrm{MS}, \mathrm{P}, \mathrm{MX}(\mathrm{Cs}), \mathrm{BJ}$

R R MS, P, BTC, MX (Cs y Sd), BJ, ZT IGF

$\mathrm{O} \quad \mathrm{R} \quad \mathrm{BE}$ I

R C BTC, MX (Cs, Mg y Sd), MS, BP, BE, BJ GI

R $\quad \mathrm{R} \quad \mathrm{BTC}, \mathrm{MS}, \mathrm{BP}, \mathrm{MX}(\mathrm{Sd}$ y Cs), BJ O

? $\mathrm{C} \quad \mathrm{VR}, \mathrm{P}$ GI

M C BP, BE, MX (Cs), BJ, MS I

M A BTC, MX (Cs, Mg y Sd), MS, P, BJ I

$? \quad ? \quad ? \quad$ IF

$\begin{array}{llll}\mathrm{R} & \mathrm{C} & \mathrm{BP}, \mathrm{BE}, \mathrm{VR}, \mathrm{BJ}, \mathrm{ZT} & \mathrm{IGF} \\ \mathrm{Pr}\end{array}$

O R MX (Mg), BE IF A

$\mathrm{R} \quad \mathrm{R} \quad \mathrm{MS}, \mathrm{BJ}, \mathrm{ZT}$ I

M C BP, BE, BJ IGF

$\mathrm{E}$

O R MS, BE, BJ IGF

M R MX (Sd, Cs), BP, BE, BJ IGF

O R BTC, P, MX (Cs, Sd), VR FI 
Turdus migratorius

Ridgwayia pinicola

\section{Mimidae}

Dumetella carolinensis

Mimus polyglottos

Toxostoma longirostre

Toxostoma ocellatum

Toxostoma curvirostre

Toxostoma crissale

Melanotis caerulescens

\section{Sturnidae}

Sturnus vulgaris

\section{Motacillidae}

Anthus rubescens

\section{Bombycillidae}

Bombycilla cedrorum

\section{Ptilogonatidae}

Ptilogonys cinereus

Phainopepla nitens

\section{Peucedramidae}

Peucedramus taeniatus

\section{Parulidae}

Vermivora peregrina

Vermivora celata

Vermivora ruficapilla

Parula superciliosa

Parula pitiayumi

Dendroica petechia

Dendorica magnolia

Dendroica coronata

Dendroica nigrescens

Dendroica chrysoparia

Dendroica virens

Dendroica townsendi

Dendroica occidentalis

Mniotilta varia

Setophaga ruticilla

Seiurus motacilla

Oporornis tolmiei

Geothlypis trichas

Wilsonia pusilla

Myioborus pictus

Myioborus miniatus

\begin{tabular}{|c|c|c|c|c|}
\hline M & $\mathrm{C}$ & BTC, MX (Mg), BP, BE, BJ & GIF & \\
\hline $\mathrm{O}$ & $\mathrm{R}$ & $\mathrm{BE}$ & $\mathrm{F}$ & $\operatorname{Pr}$ \\
\hline $\mathrm{O}$ & $\mathrm{R}$ & $\mathrm{BE}$ & IF & \\
\hline $\mathrm{R}$ & $\mathrm{C}$ & BTC, MX (Cs, Mg y Sd), MS, BE, P, BJ, & I & \\
\hline $\mathrm{O}$ & $\mathrm{R}$ & BTC, BE, MS & GIF & \\
\hline$?$ & $?$ & MS & IVG & \\
\hline $\mathrm{R}$ & $\mathrm{C}$ & $\begin{array}{l}\text { BTC, MX (Cs, Mg y Sd), MS, BP, P, BJ, } \\
\text { PA }\end{array}$ & IF & \\
\hline $\mathrm{O}$ & $\mathrm{R}$ & MS & IF & \\
\hline $\mathrm{R}$ & $\mathrm{R}$ & P, MX (Cs, Sd), MS, BTC, ZT & IF & \\
\hline
\end{tabular}

O $\quad \mathrm{R} \quad \mathrm{P}$

$\mathrm{O}$

$\mathrm{M} \quad ? \quad \mathrm{P}$

G

M R BE, MX (Sd, Cs), P, BJ

M $\quad$ C $\quad$ BP, BE, BJ, P, MX (Cs, Sd), ZT

O R MX (Cs, Mg y Sd), MS, P, BJ, PA IF

$\begin{array}{llll}\mathrm{M} & \text { ? } & \text { FI }\end{array}$

O R BP, BTC I

M C MS, BE, MX ( $\mathrm{Sd}$ y Cs), BJ, P I

M R P, MX (Cs y Sd), BTC, MS, BJ I

$\mathrm{R} \quad \mathrm{C} \quad \mathrm{BP}, \mathrm{BE} \quad \mathrm{V}$

O R BTC I

O R MS, MX (Sd), P I

$\mathrm{M} \quad \mathrm{R} \quad \mathrm{BP}, \mathrm{BE}$

M C MX (Cs y Sd), BP, BE, P, MS, BTC, BJ I

SE

M R BE, P, MS, BJ I

O R BP I

A

$\mathrm{O} \quad \mathrm{R} \quad \mathrm{BE} \quad \mathrm{I}$

M C BP, BE, MX (Sd), MS, P, BJ I

$\mathrm{M} \quad \mathrm{C} \quad \mathrm{BP}, \mathrm{BE}, \mathrm{BJ}$ I

M R BE, BJ, MS, MX (Cs, Sd), P I

? $\quad \mathrm{R} \quad \mathrm{P}$ I

$\mathrm{M} \quad$ ? $\mathrm{P}, \mathrm{VR} \quad \mathrm{I}$

M R BTC, VR, MS I

M $\quad \mathrm{C} \quad \mathrm{VR}, \mathrm{P} \quad \mathrm{I}$

M? R? C MX (Sd), BP, BE, P, BTC, MS, BJ I

$\mathrm{R} \quad \mathrm{C} \quad \mathrm{BP}, \mathrm{BE}, \mathrm{P}, \mathrm{ZT} \quad \mathrm{I}$

$\mathrm{R} \quad \mathrm{C} \quad \mathrm{BP}, \mathrm{BE}$

FI 
Basileuterus culicivorus

Basileuterus rufifrons

Icteria virens

\section{Thraupidae}

Piranga flava

Piranga rubra

Piranga olivacea

Piranga ludoviciana

Piranga bidentata

Thraupis abbas

\section{Emberizidae}

Volatinia jacarina

Sporophila torqueola

Atlapetes albinucha

Atlapetes pileatus

Arremon brunneinucha

Pipilo chlorurus

Pipilo maculatus

Pipilo fuscus

Spizella passerina

Spizella pallida

Spizella breweri

Pooecetes gramineus

Chondestes grammacus

Calamospiza melanocorys

Passerculus sandwichensis

Ammodramus savannarum

Melospiza lincolnii

Zonotrichia leucophrys

Junco phaeonotus

\section{Cardinalidae}

Cardinalis cardinalis

Pheucticus ludovicianus

Pheucticus melanocephalus

Passerina caerulea

Passerina amoena

Passerina cyanea

Passerina versicolor

Passerina ciris

\section{Icteridae}

Agelaius phoeniceus

Sturnella neglecta

$\begin{array}{cclc}\text { O } & \text { R } & \text { BP } & \text { I } \\ \text { R } & \text { C } & \begin{array}{l}\text { BP, BE, P, BTC, MC(Cs), BJ, MS, VR, } \\ \text { ZT }\end{array} & \text { I } \\ ? & ? & \text { BJ, MX (Cs) } & \text { FIG } \\ & & & \\ \text { R } & \text { R } & \text { BP, BE, P, BTC, MC (Cs, Sd), BJ, MS, VR } & \text { GIFN } \\ \text { R? } & \text { C } & \text { BTC, MX (Cs, Mg, Sd), BP, BE, BJ } & \text { NIFG } \\ \text { O } & \text { R } & \text { BTC, MX (Sd }) & \text { FI } \\ \text { M } & \text { R } & \text { BE, BTC, BJ, MX (Sd), MS } & \text { GIFN } \\ \text { R } & \text { R } & \text { BE } & \text { GIF } \\ \text { R } & \text { R } & \text { BTC, VR } & \text { FI }\end{array}$

O

\section{R}

$\mathrm{R}$

$\mathrm{R}$

$\mathrm{R}$

$\mathrm{R}$

$\mathrm{M}$

R

$\mathrm{R}$

$\mathrm{R}$

$\mathrm{M}$

M

M

M

M

$\mathrm{M}$

$\mathrm{R}$

$\mathrm{M}$

M

M

$\mathrm{M}$
$\mathrm{R}$$$
\text { R }
$$

$\mathrm{MX}(\mathrm{Mg}), \mathrm{P}$

IFN

$$
\text { R BTC, P, VR }
$$$$
\text { R BE }
$$$$
\text { R BP }
$$$$
\mathrm{R} \quad \mathrm{BP}, \mathrm{BE}
$$$$
\text { R MS, MX (Sd) }
$$
I

IG

GIFN

GIFN

GIF

FI

IFG

GFI

GFI

GFI

IG

GIF

A BP, BE, BJ, MS, ZT

BTC, MX (Cs, Mg, O y Sd), MS, BE, P, BJ, PA, ZT

IFG

IG

IG

IG

$\mathrm{O}$

G

GIF

IG

GI

IG

IFV

CE

R C BTC, MX (Cs, Mg y Sd), MS, P, BJ GFI

M R BTC IF

R C BTC, MX (Mg, Sd), BP, BE, BJ, MS, ZT, FI SE

M R BTC, MX (Mg, O y Sd), P, MS, BJ I

O R BTC, MS GIF

GIF SE

GIF

GIF

M $\quad \mathrm{R} \quad \mathrm{BTC}, \mathrm{MX}(\mathrm{Cs}, \mathrm{Sd}), \mathrm{MS}$ GIF

SE

CE

E

E

SE SE SE

$\begin{array}{llll}\mathrm{R} & \mathrm{C} & \mathrm{A}, \mathrm{P} & \mathrm{IG} \\ ? & ? & \mathrm{MS}, \mathrm{P}, \mathrm{PA} & \mathrm{IG}\end{array}$ 


\begin{tabular}{|c|c|c|c|c|c|}
\hline Xanthocephalus xanthocephalus & ? & $\mathrm{R}$ & $\mathrm{P}$ & IG & \\
\hline Quiscalus mexicanus & $\mathrm{R}$ & $\mathrm{R}$ & BTC, P & $\mathrm{O}$ & \\
\hline Molothrus aeneus & $\mathrm{R}$ & $\mathrm{C}$ & $\mathrm{P}, \mathrm{MS}, \mathrm{MX}(\mathrm{Cs}, \mathrm{Sd}), \mathrm{VR}$ & $\mathrm{O}$ & \\
\hline Molothrus ater & $\mathrm{R}$ & $\mathrm{R}$ & $\mathrm{BTC}, \mathrm{MX}(\mathrm{Mg}), \mathrm{BE}$ & IFG & \\
\hline Icterus wagleri & $\mathrm{R}$ & $\mathrm{C}$ & BTC, MX (Cs, Mg y Sd), MS, BJ & IFV & \\
\hline Icterus cucullatus & M & $\mathrm{R}$ & $\mathrm{BP}, \mathrm{P}$ & IFG & \\
\hline Icterus gularis & $\mathrm{O}$ & $\mathrm{R}$ & MS, BE & IFG & \\
\hline Icterus graduacauda & $\mathrm{R}$ & $\mathrm{R}$ & $\mathrm{MX}(\mathrm{Sd}), \mathrm{BE}, \mathrm{P}, \mathrm{MS}, \mathrm{BJ}, \mathrm{VR}$ & IFG & \\
\hline Icterus galbula & M & $\mathrm{R}$ & BTC, MX (Mg y Sd), MS, P & IFG & \\
\hline Icterus parisorum & $\mathrm{R}$ & $\mathrm{R}$ & BTC, MS, P, MX (Sd), BJ, BE & IFG & \\
\hline Psarocolius montezuma & $\mathrm{O}$ & $?$ & $\mathrm{P}$ & FVI & $\operatorname{Pr}$ \\
\hline \multicolumn{6}{|l|}{ gillidae } \\
\hline Euphonia affinis & $\mathrm{R}$ & $\mathrm{C}$ & VR, BTC & I & \\
\hline Euphonia elegantissima & $\mathrm{R}$ & $\mathrm{R}$ & BTC, BE, MS, BJ & FI & \\
\hline Carpodacus mexicanus & $\mathrm{R}$ & A & $\begin{array}{l}\text { BTC, MX (Cs, Mg y Sd), MS, BE, P, PA, } \\
\text { BJ }\end{array}$ & GF & \\
\hline Carduelis pinus & $\mathrm{R}$ & $\mathrm{R}$ & $\mathrm{BP}$ & IFG & \\
\hline Carduelis notata & $\mathrm{R}$ & $\mathrm{R}$ & $\mathrm{BP}, \mathrm{BJ}$ & IFG & \\
\hline Carduelis psaltria & $\mathrm{R}$ & $\mathrm{C}$ & BTC, MX (Mg y Sd), MS, BE, P, BJ, VR & IFG & \\
\hline Coccothraustes abeillei & $\mathrm{R}$ & $\mathrm{R}$ & $\mathrm{BE}$ & IGF & \\
\hline
\end{tabular}

\section{Passeridae}

Passer domesticus

R A P

GI 